\author{
Angela Kuck ${ }^{\mathrm{a}}$ \\ Franziska Roemer ${ }^{b}$
}

\section{Natürlicher Umgang mit \\ Pathologien im Klimakterium}

Welche Ergänzung kann die anthroposophisch erweiterte Medizin anbieten, sowohl bezüglich einer Sinngebung und qualitativen Steigerung des Lebensausdrucks im Klimakterium als auch bezüglich der Behandlung und Linderung der mit der Umstellung verbundenen Beschwerden? Wie kann es gelingen, von einer einförmigen, nur die Jugendkräfte einseitig erhalten wollenden Sichtweise überzugehen zu einer Bejahung der Umwandlung und Reifung sowie zu einer Entdeckung und Ausschöpfung der damit verbundenen individuellen Potenziale?

Das Klimakterium wird im Volksmund auch als Wechsel oder Abänderung bezeichnet. Es ist also ein Aktivum und nicht nur ein Erleiden der damit verbundenen Unannehmlichkeiten. Ein neues Gleichgewicht will gefunden und errungen werden - in leiblicher, seelischer und auch geistiger Hinsicht. Ein Übergang von einer mehr körpergebundenen zu einer körperlichen, sozialen und spirituell freieren Lebensphase findet statt. Etwas Neues möchte spürbar in das Leben eintreten; etwas, das mit dem inneren Leben zu tun hat. Das Neue ist jedoch von Frau zu Frau verschieden und kann nur selbst entdeckt und entwickelt werden. Es gibt also kein Patentrezept; vielmehr ergibt sich eine ganz individuelle Lebensfrage und damit

a Chefärztin Gynäkologie und Geburtshilfe, Paracelsus-Spital, Richterswil, Schweiz

b Praktische und Anthroposophische Ärztin, Bad Boll, Deutschland

\title{
Wenn die Kraft aus den Organen frei wird - Aufbruch zu neuen Lebenssphären
}

eine Chance zur Weiterentwicklung. Das Wohl der Familie oder der Erfolg im Beruflichen haben als alleinige Werte keinen Bestand mehr. Von dieser starken Orientierung nach aussen gilt es, Abstand zu nehmen und zunächst Inseln zu schaffen, die der Erholung und Pflege dienen sowie Freiraum schaffen für die Erkenntnis und Anerkennung der bisher im Leben erworbenen Fähigkeiten und Kompetenzen. Davon ausgehend geschieht allmählich der Aufbau einer mehr selbstbestimmten Perspektive, von Kreativität und Weiterbildung sowie der Pflege auch weiterer sozialer Kontakte. Oft befinden sich gerade in dieser Lebensphase einer Frau ihre Kinder im Stadium der Pubertät und gehen immer selbstständiger eigene Wege. Wenn die Mutter es nun schafft, ebenfalls ihre Bedürfnisse zu äussern und ihren eigenen Lebensweg zu gestalten, wirkt dies anders als zunächst befürchtet - auf die Souveränität und Eigenständigkeit der Jugendlichen positiv zurück.

\section{Gegensätzliche Konstitutionstypen im Klimakterium}

Auch wenn die während des Klimakteriums gestellten Herausforderungen und Chancen sehr individuell sind, lassen sich dennoch zwei definierbare Frauentypen unterscheiden, die polar mit der Situation umgehen und auch von unterschiedlichen Therapien profitieren.

\section{Prädominanz des Nerven-Sinnes- Systems}

Der eine Typus ist asthenisch und zierlich, eher hell an Haut und Haaren, in der Jugend zwar früh entwickelt, aber schlussendlich doch nicht stark mit den Erdenkräften verbunden. Diese Frau ist oft idealistisch, baut tüchtig und fleissig, manchmal bis zum Perfektionismus, ihre beruflichen Qualitäten aus. Ein Beispiel dafür könnte «die Oberstudienrätin» sein. Menschenkundlich hat man es mit einem einflussstärkeren NervenSinnes-System zu tun. In den Wechseljahren führt dies zu einer raschen körperlichen Erschöpfung, und die Phantasiekräfte gehen im Gefühl der Überlastung mit den bisher doch so gut bewältigten Pflichten unter. Die Hitzewallungen treten vermehrt am Tag auf, häufiger auch bei verstärkten Anforderungen von aussen, und der Schlaf wird oberflächlich und schlechter. Hier besteht die Gefahr eines zu starken Abbaus durch die Kräfte des Nerven-Sinnes-Systems, der zu Schleimhautatrophie und Osteoporose führen kann. Es gilt also für die Frau, ohne schlechtes Gewissen das Tagespensum zu reduzieren, Pausen einzuschieben und auf eigene, kürzere Rhythmen zu achten. Das bisher zu Recht Erworbene steht ja zur Verfügung und kann von Innen noch einmal ganz neu ergriffen werden.

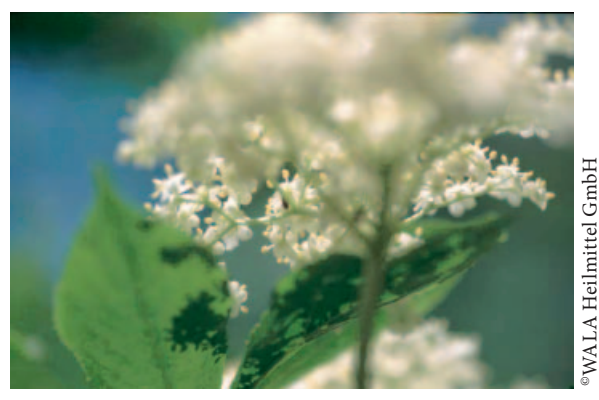

Abb. 1. Holunder (Sambucus).

\section{KARGER}

Fax +49761 4520714 Information@Karger.d www.karger.com
(๑) 2011 S. Karger GmbH, Freiburg

Accessible online at: www.karger.com/szg
Dr. med. Angela Kuck

Gynäkologie und Geburtshilfe

Paracelsus-Spital Richterswil

Bergstrasse 16, 8005 Richterswil, Schweiz

Tel. +41 44 787-2450

angela.kuck@paracelsus-spital.ch 
Prädominanz des Stoffwechselsystems Der andere Typus entspricht eher kräftigen, dunkelhaarigen, leicht adipösen Frauen, die zwar später entwickelt, aber fest mit der Erde verbunden sind. Sie sind lebenstüchtig, praktisch zupackend, können organisieren und sind als Familienmutter gerne für andere da. In den Wechseljahren leiden sie unter der Leere des Empty-Nest-Syndroms. Sich selbst vergessend, werden ihre Tätigkeiten leicht ziellos, und es besteht die Gefahr von Depressionen. Oft kommt es $\mathrm{zu}$ einer Gewichtszunahme. Hier ist die Information wichtig, dass nach den Wechseljahren der Grundumsatz um ein Viertel niedriger ist und sich die Nahrungszufuhr anpassen muss. Hitzewallungen mit reichlichem Schwitzen begleiten die Nacht, weil die Tagkräfte nicht genügend gebraucht werden: Die oberen Wesensglieder versuchen hier, in der Nacht den Leib zu individualisieren, wozu sie am Tag zu wenig Gelegenheit haben. Eine Osteoporose entwickelt sich selten, da aus den Androgenen des Fettgewebes zusätzlich Östrogene freigesetzt werden. Hier ist der Schwerpunkt ein anderer: Es gilt, tagsüber für die Frau stimmige Aktivitäten zu fördern - in beruflicher, sportlicher sowie sozialer Hinsicht. Die Erfahrungen in der Familie sind hierfür die beste Voraussetzung. Ein einprägsames Bild für diese Frau könnte die «italienische Mutter» sein. Menschenkundlich liegt eine Prädominanz des Stoffwechselsystems vor, mit dem die höheren Wesensglieder zu stark, aber in starrer und unbeweglicher Weise verbunden sind.

\section{Fazit}

Therapeutisches Ziel ist, die Empfindungs- und Ich-Organisation beim Nerven-Sinnes-Typus stärker mit dem Stoffwechselsystem in Verbindung zu halten, während beim StoffwechselTypus die höheren Wesensglieder stark leibgebunden sind und aus ihrer Starre befreit und individualisiert werden müssen.

In jedem Fall gilt es, die rhythmischen Prozesse durch freie künstlerische Betätigung und anthroposophisch erweiterte künstlerische Therapie anzuregen, insbesondere durch die Heileurythmie den Leib zu ergreifen und durchzugestalten sowie in der Seele das innere Licht zu pflegen. Mithilfe der folgenden Arzneimittel gelingt es in den allermeisten Fällen, klimakterische Beschwerden in einem gut erträglichen Rahmen zu halten.

\section{Behandlungsmöglichkeiten}

\section{Sambucus comp.}

Strukturiert den Flüssigkeitsorganismus bei klimakterischen Störungen mit Hitzewallungen und übermässiger, vor allem nächtlicher Schweisssekretion. Sambucus nigra (Holunder; Abb. 1) mit seinen durchlufteten, intensiv duftenden Blütenschirmen hat eine stark sulfurische Wirkung und ist Vorbild für die Beherrschung des ausufernden Flüssigkeitsprozesses durch die Empfindungsorganisation.

\section{Ovaria comp.}

Reguliert und harmonisiert die endokrine ovarielle Umstellung und sorgt für einen weniger abrupten, stufenloseren Übergang. Die ersten hormonellen Veränderungen und Instabilitäten beginnen im Durchschnitt 7 Jahre vor der Menopause: Durch die intensivere hypophysäre Stimulation reagieren die Ovarien zunächst mit einer Hyperöstrogenämie bei relativem Progesteronmangel. Die Blutungen können früher eintreten und stärker werden - bei gleichzeitiger Aufgedunsenheit, körperlichem und seelischem Schweregefühl -, bevor sie zu einem späteren Zeitpunkt seltener und schwächer werden und schliesslich sistieren. Nach der Menopause nimmt die hypophysäre Überreaktion die Form von unrhythmischen, wechselnd starken pulsatilen $\mathrm{LH}$ (luteinisierendes Hormon)- und FSH(follikelstimulierendes Hormon)-Peaks mit gleichzeitig bestehender Sympa-

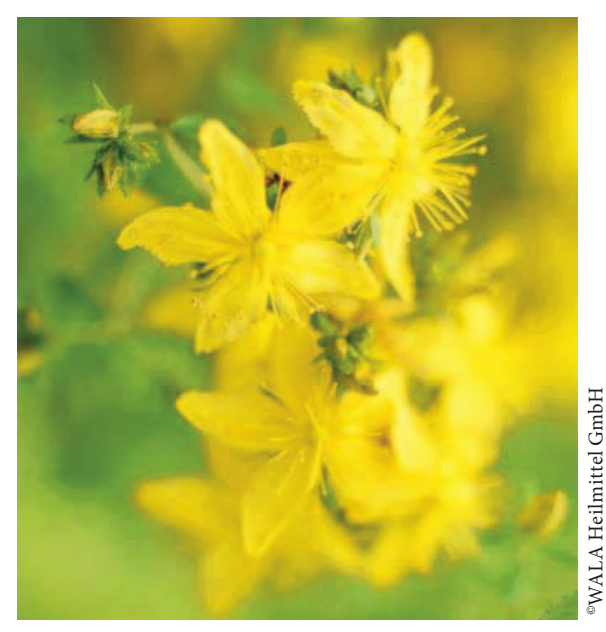

Abb. 2. Johanniskraut (Hypericum).

Tab. 1. Ausgewählte Arzneimittel zur Therapie von Pathologien im Klimakterium

\begin{tabular}{ll} 
Krankheitssituation & Empfohlenes Arzneimittel \\
\hline $\begin{array}{l}\text { Hitzewallungen im Klimakterium } \\
\text { Allgemeine Regulation der hormonellen Umstellung im Klimakterium }\end{array}$ & Sambucus comp. \\
$\begin{array}{l}\text { Nervös gereizte Verstimmung im Klimakterium } \\
\text { Depressive Verstimmung }\end{array}$ & Melissa/Sepia comp. \\
$\begin{array}{l}\text { Hysteriforme Verstimmung } \\
\text { Schilddrüsenfunktionsstörungen im Klimakterium }\end{array}$ & Aurum/Apis regina comp. \\
$\begin{array}{l}\text { Myome } \\
\text { Senkungsbeschwerden }\end{array}$ & Ignatia comp. \\
Chronisch-entzündliche und dystrophische Veränderungen & Spongia/Aurum/Pulsatilla comp. \\
am äusseren Genitale & Berberis/Sepia comp. \\
\hline
\end{tabular}


thikotonie an, die zeitlich parallel $\mathrm{zu}$ den Hitzewallungen verlaufen. Ovaria comp. reguliert allgemein diese hormonellen Veränderungen und gleicht vor allem die perimenopausalen Östrogen-Unregelmässigkeiten aus. Insofern ist Ovaria comp. ein allgemeines Mittel bei klimakterischen Störungen. Die Dosierung kann zwischen 1- bis zu 5-mal täglich 5-10 Globuli velati variieren. Viele Frauen spüren selbst, welche Dosis sie benötigen: Diese kann zu Beginn des Klimakteriums eine mittlere Höhe betragen, dann ansteigen, um schliesslich wieder allmählich abzufallen. Zusätzliche Injektionen von Ovaria comp. 1- bis 3-mal wöchentlich verstärken die Wirkung. Besonders die Nerven-Sinnes-betonte Frau benötigt diese funktionelle hormonelle Unterstützung. Im Falle starker organischer Abbauerscheinungen und besonders bei einer frühen Menopause (vor dem 40. Lebensjahr) sowie bei einer starken kräftemässigen Beanspruchung ist nichts prinzipiell gegen eine vorübergehende stoffliche hormonelle Unterstützung einzuwenden. Studien (Woman's Health Initiative (USA) und Million Women Study (UK)) zeigen, dass die unerwünschten Effekte der Hormonersatztherapie erst nach einer Einnahmezeit von 4 Jahren signifikant nachweisbar werden.

\section{Melissa/Sepia comp.}

Harmonisieren das Eingreifen von Ich- und Empfindungsorganisation im Klimakterium. Die verstärkte relative Ablösung von Ich- und Empfindungsorganisation aus den Reproduktionsorganen hat eine stärkere Befreiung in das Seelische zur Folge, wobei die höheren Wesensglieder für eine gewisse Zeit sozusagen «vagabundieren», bevor sie ein neues leiblich-seelisches Gleichgewicht finden. Zum Aufbau dieses neuen Gleichgewichts trägt die seelisch-geistige Betätigung der Frau wesentlich bei: Es ist statistisch gesichert, dass Frauen mit dem Empty-Nest-Syndrom am meisten unter den Wechseljahren lei- den. Weniger Beschwerden haben berufstätige Frauen, noch weniger jene Frauen, die in diesen Jahren bewusst einen inneren oder äusseren Neuanfang durchleben. Es ist bezeichnend, dass afrikanische Frauen, die mit der Menopause in den Ältestenrat kommen und dort sinnvoll ihre Erfahrungen einbringen, über keinerlei Wechseljahrbeschwerden klagen. Melissa/ Sepia comp. hilft bei nervös gereizter, wechselnder und erschöpft depressiver, sich selbst überfordernder Stimmung, die in der Anfangsphase bei Stoffwechselbetonung und generell bei überstarken Nerven-Sinnes-Kräften vorkommen kann.

\section{Aurum/Apis regina comp.}

Aurum chloratum und Hypericum perforatum (Johanniskraut; Abb. 2) stärken das eigene seelische Licht, Acidum phosphoricum und Apis regina lindern die Erschöpfung, Strychnos ignatii (Ignatiusbohne) und Avena sativa (Hafer) gleichen die Stimmung aus. Insofern hilft Aurum/ Apis regina comp. allgemein bei manifester und larviert depressiver Symptomatik im Klimakterium. Dazu gehören Schwindelerscheinungen, Kopfschmerz, Konzentrationsstörungen und Gedächtnisschwäche, Stimmungsschwankungen, Erschöpfung, Magenbeschwerden sowie Kreuzschmerzen.

\section{Ignatia comp.}

Enthält Bryophyllum sowie Lachesis muta und bessert psychische klimakterische Störungen, besonders hysteriformer Art beim StoffwechselTypus. Kennzeichnend für diese Indikation ist viel und unpräzises Reden, zu starke Selbstbezogenheit sowie Widersprüchlichkeit.

\section{Spongia/Aurum/Pulsatilla comp.}

Bei klimakterischen Beschwerden mit Schilddrüsenfunktionsstörungen und damit möglicherweise einhergehender Herzbeteiligung: Die sich aus den Unterleibsorganen lösenden höheren Wesensglieder können gelegentlich

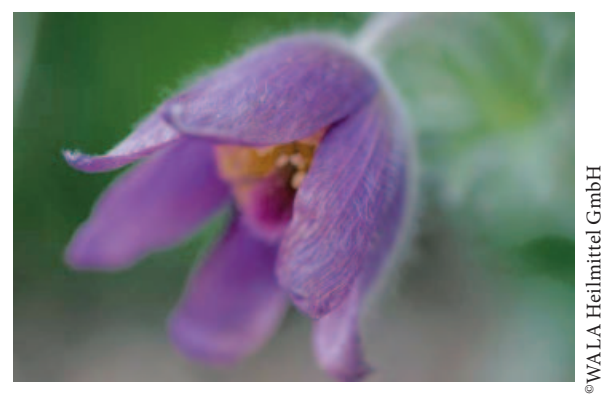

Abb. 3. Küchenschelle (Pulsatilla).

geradezu hinaufschlagen und $\mathrm{zu}$ Funktionsstörungen im mittleren und am Übergang zum oberen Menschen führen (Küchenschelle; Abb. 3).

\section{Berberis/Sepia comp., Globuli velati} Fördern die Rückbildung von Myomen und Blutungsstörungen im Klimakterium (Sauerdorn; Abb. 4).

Prunus spinosa e floribus W5\%, Oleum Für Öldispersionsbäder besonders beim ersten Typus zur Anregung des Aufbaustoffwechsels bei nervöser Erschöpfung, Asthenie und vegetativer Dystonie. Auch bei der Auswahl persönlicher Konstitutionsmittel kann man sich für eine erste Orientierung gut dieser Typengliederung bedienen: Sepia, die Säuremittel und Cimicifuga gehören zum Nerven-Typus (Wallungen eher von oben nach unten), während Lachesis, Sulfur und Sanguinaria zum Stoffwechsel-Typus passen (Wallungen eher von unten nach oben).

\section{Senkungsbeschwerden}

Dem Zurückziehen der höheren Wesensglieder kann man entgegenwirken, indem man sozusagen ein inneres Gefühl für die Unterleibsorgane entwickelt und diese im ganzen Menschen integriert hält. Dazu können einige Stunden bei einer guten Physiotherapeutin beitragen. Es geht darum, den eigenen Beckenboden mit der Empfindung kennen zu lernen und ihn mit einem adäquaten Spannungszustand (Eutonus) zu durchdringen. Ein Hypotonus führt zu Erschlaffungszuständen, organischen Senkun- 
gen und einem Rückgang der Vitalität, während ein - unter Umständen angstbegleiteter - Hypertonus Verkrampfungen, schmerzhafte Myotonien bis hin zur spastischen Obstipation zur Folge hat. Der Beckenboden soll also aktiviert werden, bei verschiedenen Bewegungen, wie z.B. beim Gehen, locker gespürt und bei anstrengenden Tätigkeiten, wie Schieben usw., nach Möglichkeit nicht belastet werden. Zu häufiges Wasserlassen sollte man sich erst gar nicht angewöhnen, da dies der Urge-Komponente Vorschub leistet. Eine gezielte Beckenbodengymnastik und Heileurythmie können auf dieser Grundlage erlernt werden.

\section{Senecio comp.}

Wirkt einerseits durch die Kupferpotenz einer Verkrampfung entgegen und fördert andererseits durch die Stannum-Potenz den Eutonus. Die Bänder und Faszien werden vitalisiert und gestrafft, die plastische Organgestalt bleibt erhalten. Die Dosierung beträgt 1- bis 3-mal täglich 10 Globuli velati.

\section{Solum uliginosum comp. Oleum}

Einreibungen von Kreuz, Damm und Hüftgegend wirken durchwärmend und lösend.

\section{Citrus, Oleum aethereum 10\%}

$\mathrm{Zu}$ Öldispersionsbädern bei Bindegewebsschwäche, Senkungen und Varikosis.

\section{Chronisch-entzündliche und dystrophische Veränderungen am äusseren Genitale}

Die Intimgegend und der HautSchleimhaut-Übergang bedürfen mit zunehmendem Alter einer besonderen Pflege. Im Klimakterium muss auch in dieser Zone ein neues Gleichgewicht gefunden werden.

Rosmarinus/Prunus comp., Gelatum Fördert die Regenerationsvorgänge bei chronisch-entzündlichen und dystrophen Veränderungen im äusseren

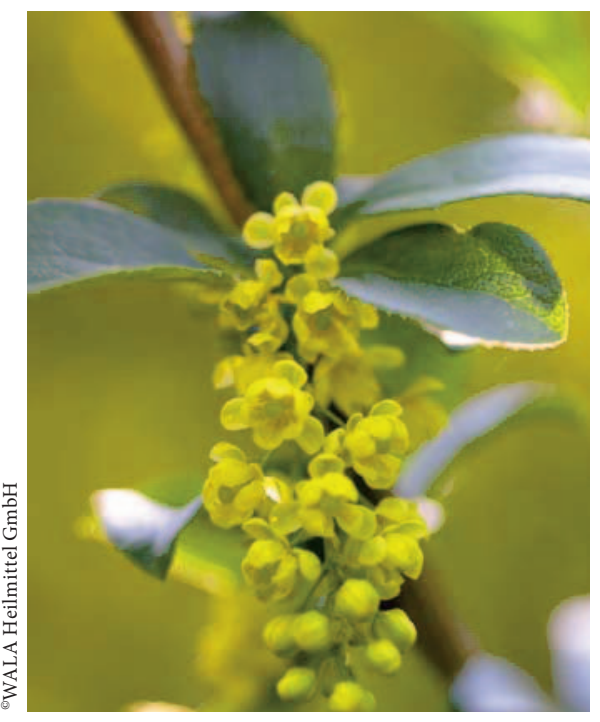

Abb. 4. Berberis (Berberis vulgaris).

Genitalbereich. Hierzu gehören chronisch-entzündliche Prozesse auf dem Boden einer beginnenden oder bestehenden Atrophie sowie dystrophe Veränderungen, beispielsweise Kondylome. Lindert Pruritus. Rosmarinus/Prunus comp., Gelatum soll nicht in die Scheide eingeführt werden. Bei Kolpitiden, auf infektiöser oder trockener Grundlage, ist das mildere Majorana comp., Gelatum angezeigt.

\section{Fazit}

Die anthroposophische Menschenkunde stellt die klimakterischen Einzelsymptome in einen grösseren Sinnzusammenhang. Das StoffwechselReproduktions-Gliedmassen-System ist einerseits Polarität des Nerven-Sinnes-Systems und darf nicht zu stark von seiner abbauenden Geste überwältigt werden. Andererseits sind von der Neuorientierung der Wesensglieder auch die Bereiche der Ernährung und des Stoffwechsels sowie die körperliche Bewegung und Stabilität betroffen. Die Aktivität von Empfindungs- und Ich-Organisation, die sich vermehrt aus den unteren Lebensprozessen lösen, kann willentlich ergriffen werden. Da sich der physiologische Grundumsatz um rund ein Viertel reduziert, bedarf die Ernährung vor allem beim Stoffwechsel-Typus der Umstellung. Eine leichtere, obst- und gemüsereiche, an Kohlenhydraten und gesättigten Fetten ärmere Ernährung ist bekömmlicher. Die Nerven-Sinnesbetonte Frau dagegen zeigt teilweise eine zu stark abbauende Tendenz im gesamten Stoffwechsel und verliert sogar an Gewicht. Hier sind Zusatzmahlzeiten, Milchspeisen mit normalem Fettgehalt und gutes Olivenöl wichtig. Bitterstoffe, wie sie im Gentianae comp. Extractum aquosum und Bitter Elixier enthalten sind, stärken die Verdauungskraft und erlauben grössere Mahlzeiten. Auch der Bereich der körperlichen Bewegung will neu bewusst wahrgenommen und gepflegt werden. Die zierliche, erschöpfte Frau profitiert oft von der Heileurythmie, während die kräftigere, vollblütigere Frau mehr in der sportlichen Bewegung, z.B. durch regelmässige Gymnastik, gefordert werden kann. Spaziergänge an der frischen Luft in einer schönen Umgebung beleben das rhythmische System und sind in jedem Fall günstig. Auch zur Osteoporoseprophylaxe eignet sich aktive körperliche Bewegung im Licht am besten, denn die Stabilität der Knochenbälkchen und die Intensität der Verknöcherung und Mineralisierung steht in direkter Abhängigkeit von der körperlichen Belastung sowie den Druck- und Zugkräften. Statistische Untersuchungen belegen beispielsweise, dass regelmässige isometrische Übungen die Frakturrate stärker verringern als eine Hormonersatztherapie. Der Bambus ist hier eine vorbildliche Heilpflanze, da er starke Substanzbildungskräfte mit intensiver Mineralisierung bei elastischer Beweglichkeit verbindet. Phyllostachys e nodo D3, Globuli velati regt die Organisations- und Gestaltungskräfte an, vor allem bei degenerativen Wirbelsäulenerkrankungen und bei Bindegewebsschwäche. In jedem Fall ist es wichtig, das Augenmerk der Frau während der Wechseljahre auf die neuen seelisch-geistigen Perspektiven zu lenken, die sich aus diesen physiologischen Vorgängen ergeben, da sich eine schöpferische und initiative Haltung auch wieder positiv auf die Gestaltung der Organe auswirkt. 